\title{
Bilan des études expérimentales de transferts de technétium à des sédiments et à des espèces marines benthiques et comparaison à des résultats in situ
}

\author{
G. APROSI ${ }^{\star}, M . M A S S O N^{\star \star}$ \\ (Manuscrit reçu le 16 mars 1983)
}

\begin{abstract}
RÉSUMÉ
Le technétium (TC), radioélément à vie longue ( $T p=2,1 \times 10^{5}$ ans) n'existe pas à l'état naturel et provient principalement des opérations liées au cycle des combustibles nucléaires. Le manque de données en ce qui concerne les transferts du technétium à l'écosystème a été à l'origine de nombreuses études de laboratoire.

Le technétium se fixe sur les sédiments beaucoup plus en milieu anoxique et réducteur qu'en milieu oxydant; les valeurs du coefficient de distribution varient de 0 en milieu oxydant à 1000 en milieu réducteur. Quelle que soit la voie de contamination, l'élimination se fait selon deux phases : une phase rapide de quelques jours et une phase lente. Le technétium est particulièrement retenu par des organes qui jouent un rôle régulateur (foie, glande digestive...) et épurateur (rein...) ; le muscle le fixe peu. Certains paramètres comme la lumière chez les algues, la forme physico-chimique chez les organismes ont une influence ; au contraire la taille, la salinité et la température n'en ont pas. Les analyses des teneurs en ${ }^{90} \mathrm{Tc}$ chez les espèces prélevées autour des usines de retraitement de La Hague et de Windscale confirment les résultats expérimentaux. Le milieu marin étant essentiellement oxydant, le technétium se fixera peu et présentera une grande capacité de diffusion.
\end{abstract}

\section{ABSTRACT}

To obtain basic information for the evaluation of the radiological impact of technetium (Tc) on the marine environment, investigations are performed by different laboratories. Technetium is not a natural element and the main source of production is the nuclear fuel cycle. Under anoxic conditions, in presence of reducing sediments, the distribution coefficients are very high $\left(K_{D}=10^{3}\right)$.

* Electricité de France, Direction des études et recherches, Service "applications, électricité, environnement", 6, quai Watier, 78400 Chatou.

** Commissariat à l'énergie atomique, Institut de protection et de sûreté nucléaire, Département d'études et de recherches en sécurité, Laboratoire de radioécologie marine, BP 270, 50107 Cherbourg. 


\begin{abstract}
Concentration factors from water to species are mostly very low (FC 1 to 10); however, concentration factors up to 1000 have been observed for a few species such as macrophytic brown algae, worms and lobster. Biochemical analysis shows that Tc is bound with protein. The transfer factors between sediment and species are very low (FT $<0,5)$. The biological half-life (Tb) was determined in some marine organisms which had accumulated the radionuclide from water-contamined food or from sediments. The loss is biphasic in storage organs (liver and kidney) ; uptake in the edible parts is low. Among the parameters studied (light for algae, physico-chemical form of Tc, salinity and temperature). Only light and the physico-chemical forms have an effect on the accumulation of technetium.
\end{abstract}

Analyses of ${ }^{99} \mathrm{Tc}$ concentrations in species collected near the La Hague and Windscale (Sellafield) reprocessing plants confirm the experimental studies. Since sea water is likely to be an oxydant environment, technetium appears as a conservative element.

\title{
1. INTRODUCTION
}

Le technétium (Tc) est un des radioéléments à vie longue susceptible d'être présent dans le milieu marin principalement à cause des rejets des usines de retraitement, le stockage des déchets pouvant ultérieurement poser un problème. Ce radioélément n'existe pas à l'état naturel et son comportement dans la biosphère était inconnu jusqu'à la dernière décade, période pendant laquelle des études ont été engagées par plusieurs laboratoires (1). En France, le Laboratoire de radioécologie marine (LRM) de La Hague (Commissariat à l'énergie atomique) avec une contribution de la Direction des études et recherches d'Electricité de France (EF) s'est intéressé au transfert de technétium en milieu marin à partir de l'eau, des sédiments et de la nourriture pour diverses espèces [3-7, 37]. Les radioisotopes du technétium sont nombreux (nombre de masse du noyau de 92 à 105), mais ce sont les ${ }^{99} \mathrm{Tc}$ (émetteur $\beta$, période physique $\mathrm{T}_{\mathrm{p}}=2,1 \times 10^{5} \mathrm{ans}$ ) et $99 \mathrm{mTc}$ (émetteur $\gamma, \mathrm{T}_{p}=6 \mathrm{~h}$ ) que l'on étudiera particulièrement car, seuls, ils sont rejetés dans l'environnement. Le ${ }^{99} \mathrm{Tc}$ doit être utilisé à une concentration massique dans l'eau de l'ordre de $10^{-3} \mathrm{ppm}$ pour être facilement mesuré ; cette concentration est de plusieurs ordres de grandeur supérieure à celle du ${ }^{99} \mathrm{Tc}$ in situ trouvé, par exemple, au large de La Hague $\left(\simeq 3 \times 10^{-10} \mathrm{ppm}\right)$. C'est la raison pour laquelle les études expérimentales ont été réalisées le plus souvent avec le traceur $95 \mathrm{~m}$ Tc qui permet d'avoir des concentrations massiques proches de celles in situ et qui, en tant qu'émetteur $\gamma$, permet de pratiquer des essais non destructifs et de suivre une cinétique.

Une synthèse sera faite ici des principaux résultats obtenus par les divers laboratoires qui ont travaillé sur ce sujet. Un rapport technique plus détaillé est en cours de rédaction.

(1) Laboratoire international de radioécologie marine (ILMR), IAEA, Monaco ; Laboratoire pour l'étude du milieu marin (ENEA), Santa Teresa, La Spezia (Italie); Laboratoire d'océanographie de l'Université d'Etat de l'Orégon (USA); Laboratoire de radiobiologie du ministère de l'agriculture (MAFF), Lowestoft (UK). 


\section{GÉNÉRALITÉS}

\subsection{Sources de technétium}

II y a trois sources possibles : les retombées des explosions nucléaires $\left({ }^{99} \mathrm{Tc}\right)$, les utilisations médicales $\left({ }^{99 \mathrm{~m}} \mathrm{Tc}\right)$ et le cycle du combustible nucléaire $\left({ }^{99} \mathrm{Tc}-{ }^{99 m} \mathrm{Tc}\right)[13,36,45,53]$.

En ce qui concerne les retombées des explosions nucléaires, la quantité totale de ${ }^{99} \mathrm{Tc}$ est de l'ordre de $1000 \mathrm{Ci}\left(37 \times 10^{12} \mathrm{~Bq}\right)$; quelques prélèvements d'eau de pluie effectués aux USA présentent des teneurs en ${ }^{99} \mathrm{Tc}$ de $0,14 \times 10^{-2}$ à $1,7 \times 10^{-2} \mathrm{pCi} / \mathrm{l}\left(63 \times 10^{-5} \mathrm{~Bq} / \mathrm{l}\right)[19]$.

Les utilisations médicales du ${ }^{99 \mathrm{~m}} \mathrm{Tc}$ à partir du générateur ${ }^{99} \mathrm{Mo}$ se généralisent. SAAS [45] estime à $1000 \mathrm{Ci}$ l'utilisation annuelle de ${ }^{99 m} \mathrm{Tc}$ en France, mais les quantités de ${ }^{99} \mathrm{Tc}$ qui en découlent par filiation sont très faibles, de l'ordre de $3 \times 10^{-6} \mathrm{Ci} / \mathrm{an}\left(11 \times 10^{3} \mathrm{~Bq} / \mathrm{an}\right)$.

Au cours du cycle du combustible nucléaire le ${ }^{99} \mathrm{Tc}$ est présent dans les effluents liquides des centrales nucléaires, des usines d'enrichissement et de retraitement et lors du stockage des déchets. Dans les effluents liquides des centrales nucléaires, c'est ${ }^{99 m}$ Tc qui est comptabilisé. L'activité en ${ }^{99} T c$ effectivement rejetée est considérée comme négligeable car le rapport d'activité de ces isotopes est l'inverse du rapport de leur période $\left(3 \times 10^{8}\right)$. Dans les effluents liquides des usines d'enrichissement en France, on ne possède pas de chiffre sur la quantité de technétium rejetée mais, à titre d'exemple, l'usine d'enrichissement d'uranium de Capenhurst (UK) a rejeté en 1978,1979 et 1980 des teneurs respectives en technétium de 0,062 $\mathrm{Ci}(0,0023 \mathrm{TBq}), 0,32 \mathrm{Ci}(0,0118 \mathrm{TBq}), 0,312 \mathrm{Ci}(0,0115 \mathrm{TBq})[28-30]$. Les effluents des usines de retraitements sont, actuellement, la source principale de ${ }^{99}$ Tc. Ainsi, à La Hague (France) ou à Windscale (UK), dans I'hypothèse d'une quantité nominale de 1600 t de combustibles traités par an, ce sont $1280 \mathrm{~kg}$ de ${ }^{99} \mathrm{Tc}$ qui transiteront sur chacun de ces sites. Les données concernant les rejets actuels sont fragmentaires. A Windscale, par exemple, pour les années 1978 et 1979 , on a respectivement $4839 \mathrm{Ci}$ et $1175 \mathrm{Ci}\left(1792 \times 10^{11}\right.$ et $\left.435 \times 10^{11} \mathrm{~Bq}\right)$ de ${ }^{99} \mathrm{Tc}$ [36]. A La Hague, l'étude récente de Patti sur les effluents liquides donne des teneurs de $4,7 \mathrm{à} 23 \mathrm{mCi} / \mathrm{m}^{3}\left(1,7 \times 10^{8}\right.$ à $\left.8,5 \times 10^{8} \mathrm{~Bq} / \mathrm{m}^{3}\right)$ de ${ }^{99}$ Tc dans les effluents avant rejet [41]. Le stockage' des déchets de haute activité sous forme de dépôt ou d'enfouissement dans les grands fonds océaniques est aussi une source potentielle de ${ }^{99} \mathrm{Tc}$.

Le tableau I présente une estimation de la production de ${ }^{99} \mathrm{Tc}$ à l'échelon mondial et à l'échelon de la production nationale américaine.

TABLEAU ।

Estimation de la production cumulée de ${ }^{99} \mathrm{TC}$

\begin{tabular}{|l|c|r|r|r|r|}
\hline \multicolumn{1}{|c|}{ Production } & Poids & \multicolumn{2}{c|}{ Activité } & \multirow{2}{*}{ Année } & \multirow{2}{*}{ Référence } \\
\hline Mondiale & 10 & $1,7 \times 10^{5}$ & $63 \times 10^{14}$ & 1980 & {$[33]$} \\
Mondiale & $\simeq 325$ & $5,6 \times 10^{6}$ & $2 \times 10^{17}$ & 2000 & {$[25]$} \\
USA & $\simeq 170$ & $2,9 \times 10^{6}$ & $1 \times 10^{17}$ & 2000 & {$[16]$} \\
$\cdot 1 \mathrm{~Bq}=27 \mathrm{pCi}$ & & & & & \\
\hline
\end{tabular}




\subsection{La chimie du technétium}

La chimie du technétium a fait l'objet de nombreux travaux [2, 32-34, 50]. Comme c'est le cas pour la plupart des éléments à valences multiples, les espèces chimiques du technétium (7 états de valence possibles) présentes dans les milieux aqueux seront fonction des relations complexes s'établissant entre trois types d'équilibres chimiques comme l'oxydo-réduction, l'hydrolyse, la complexation ainsi que des processus comme la formation de colloïdes et la polymérisation [40]. La forme stable et soluble du technétium dans un milieu de force ionique élevée comme le milieu marin bien oxygéné est le pertechnétate $\mathrm{TcO}_{4}{ }^{-}$(valence + ) sous forme de sels de sodium, de potassium ou d'ammonium [7, 9, 24, 35, 40].

\section{3. ÉTUDE DU TRANSFERT EAU-SÉDIMENTS}

\section{Fixation}

\section{Fixation et désorption}

Les sédiments ou les suspensions constituent souvent un piège pour leśs radioéléments dans le milieu marin ; il convenait de vérifier s'il en est ainsi pour le technétium. Le tableau II résume les résultats des diverses études faites en exprimant les fixations en termes de coefficient de distribution $\left(\mathrm{K}_{\mathrm{D}}\right)$.

\section{TABLEAU II}

Valeurs expérimentales des $K_{D}$ * du technétium en milieu marin

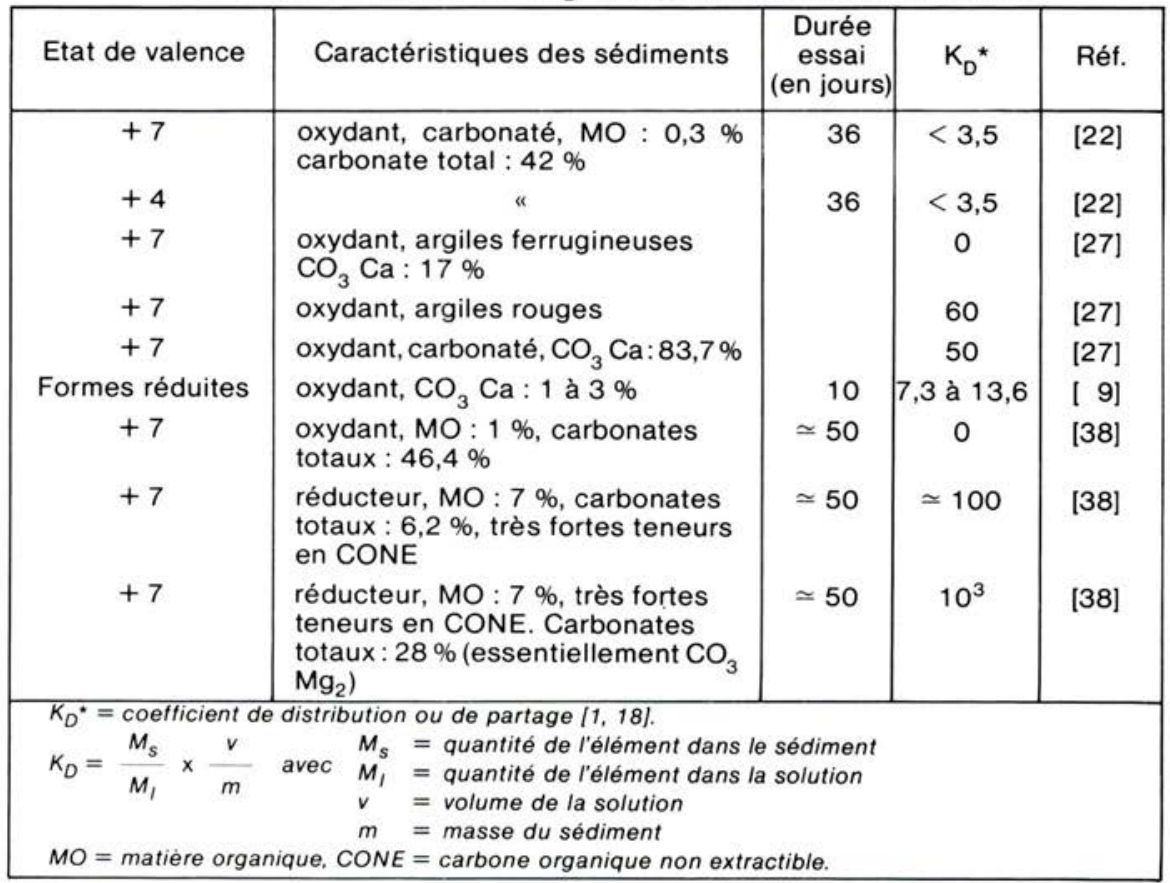


D'une manière générale, l'ion pertechnétate $\left(\mathrm{TcO}_{4}{ }^{-}\right)$se fixe peu ou pas sur les sédiments. Pour les sédiments oxydants, le technétium ne se fixe pratiquement pas quelle que soit la forme physico-chimique $[10,22]$, en revanche, pour les sédiments riches en matières organiques et réducteurs, divers travaux ont montré que les $\mathrm{K}_{\mathrm{D}}$ peuvent atteindre $10^{3}[38]$.

\section{Désorption}

Les études de désorption de sédiments contaminés par du technétium donnent deux types de résultats :

- Si le sédiment est de nature réductrice, le technétium est fortement retenu sous forme de sulfure ou de composés difficilement solubles $\left(\mathrm{TcO}_{2}\right)$.

- Si le sédiment est oxydant, les liaisons sont très faibles et le technétium s'élimine très rapidement dans l'eau circulante. L'équipe de I'ILRM, Monaco, a calculé une période d'élimination de 4,5 jours pour un tel sédiment [22].

\section{4. ÉTUDE DU TRANSFERT DU TC A DES ESPĖCES MARINES BENTHIQUES}

(Fixation et élimination)

Le mode expérimental permet d'étudier les trois voies de transfert d'un radioélément à des espèces marines : par l'eau, par la nourriture, par le sédiment. Les résultats s'expriment en termes de facteurs de transfert (FT) ou de facteurs de concentration (FC) et de période biologique (Tb). Les FC ou FT constituent la formulation la plus utilisée pour exprimer le pouvoir d'accumulation d'un organisme vis-à-vis d'un support contaminé et n'ont de sens précis que dans un schéma expérimental donné. La période biologique permet de connaître le taux d'épuration d'un contaminant par l'organisme.

\subsection{Transferts par l'eau}

\section{Fixation}

Le tableau III donne pour différentes espèces marines, les valeurs des facteurs expérimentaux avec les références bibliographiques correspondantes.

Les végétaux et animaux ont été choisis en fonction de leur abondance locale et de leur rôle dans les chaînes trophiques (consommation par l'homme par exemple). 
TABLEAU III

Facteurs de concentration (FC) expérimentaux pour quelques especces marines benthiques

\begin{tabular}{|c|c|c|}
\hline Espèces & FC & Références \\
\hline $\begin{array}{l}\text { Phytoplancton } \\
\text { Algues brunes } \\
\text { Algues rouges } \\
\text { Algues vertes } \\
\text { Annélides (vers de sable) } \\
\text { Annélides (vers de vase) } \\
\text { Echinodermes (oursins) } \\
\text { Poissons } \\
\text { Mollusques } \\
\text { Gastéropodes } \\
\text { Ormeau } \\
\text { Patelle } \\
\text { Pieuvre } \\
\text { Lamellibranches } \\
\text { Moule } \\
\text { Huître } \\
\text { Coquille St Jacques } \\
\text { Coque } \\
\text { Palourde } \\
\text { Crustacés } \\
\text { Crevette } \\
\text { Tourteau } \\
\text { Crabe vert } \\
\text { Araignée de mer } \\
\text { Gammare } \\
\text { Langouste } \\
\text { Cigale de mer } \\
\text { Homard }\end{array}$ & 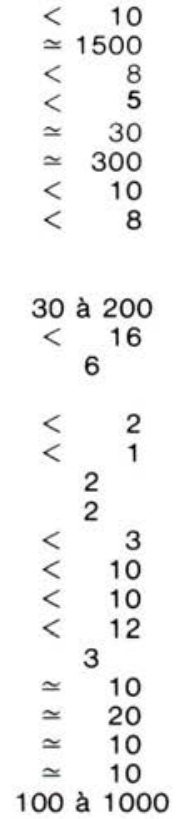 & $\begin{array}{l}{[7-12-51]} \\
{[6]} \\
{[47]} \\
{[6-11-21]} \\
{[6-11]} \\
{[6]} \\
{[6]} \\
{[6-22]} \\
{[6-21-47-48]} \\
{[6-21-47-48]} \\
{[6-10]} \\
{[6]} \\
{[6]} \\
{[10-38]} \\
{[47]} \\
{[47]} \\
{[6-38-44]}\end{array}$ \\
\hline
\end{tabular}

Les résultats sont très différents suivant les espèces : pour les crustacés, par exemple, les facteurs de concentration sont en général inférieurs à 10, sauf pour le homard où il est de l'ordre de 1000 . Ce phénomène est pour l'instant inexpliqué, aussi des études sur la localisation des sites de fixation sont entreprises actuellement, les premiers résultats montrent l'affinité protéinique du technétium [21, 37, 38, 47, 48]. Pour les facteurs de concentration élevés on constate une divergence dans les résultats selon l'isotope. Pour le homard, les valeurs proches de 100 correspondent à l'utilisation du ${ }^{99} \mathrm{Tc}$, celles proches de 1000 correspondent au ${ }^{95 \mathrm{~m}} \mathrm{Tc}$; ceci est imputable à l'action massique du ${ }^{99}$ Tc qui pour être facilement mesuré nécessite des concentrations dans l'eau de 2,9 $\times 10^{-3} \mathrm{ppm}$ alors que in situ elles sont de l'ordre de $10^{-10} \mathrm{ppm}$ et correspondent à celles du ${ }^{95 \mathrm{~m}} \mathrm{Tc}$ lors des essais. Ce sont les organes régulateurs (foie des poissons, glande digestive des mollusques) et épurateurs (rein) qui accumulent le plus le technétium $[21,47,48]$. Par exemple, I'hépatopancréas du homard présente un facteur de concentration de 16000 alors que l'animal entier a un facteur de concentration de 1000 [38]. 
Un certain nombre de paramètres vont jouer un rôle dans la fixation, la forme physico-chimique, par exemple. Si l'on introduit dans le milieu des formes réduites ou oxydées, le résultat varie en fonction des espèces $[7,10,21]$.

D'autres paramètres - la taille, la température et la salinité - ne jouent pas un rôle dans la fixation pour les quelques espèces étudiées $[6,7,10,21]$.

\section{Elimination}

Si l'on place les espèces contaminées en milieu non contaminé, la radioactivité va s'éliminer suivant des cinétiques dont les courbes ont l'allure d'une somme d'exponentielles décroissantes. Cela correspond à la combinaison de plusieurs mécanismes ayant des cinétiques différentes. Comme pour la plupart des radioéléments, l'élimination du technétium se fait principalement selon deux phases : une phase rapide de quelques jours et une phase lente [48]. Nous présentons dans le tableau IV les périodes biologiques (Tb) de la phase lente d'élimination avec, lorsqu'on le connaît, le pourcentage d'élimination correspondant.

TABLEAU IV

Période biologique du technétium pour quelques espèces marines benthiques et fraction éliminée (\%) correspondante (la voie de contamination est l'eau)

\begin{tabular}{|c|c|c|c|}
\hline Espèce & Fraction éliminée (\%) & Tb (jours) & Références \\
\hline $\begin{array}{l}\text { Algues brunes } \\
\text { Fucus Serratus } \\
\text { Mollusques } \\
\text { Ormeau } \\
\text { Palourde } \\
\text { Moule } \\
\text { Huître } \\
\text { Echinodermes } \\
\text { Oursin } \\
\text { Poissons } \\
\text { Annélides } \\
\text { Vers de vase } \\
\text { Vers de sable } \\
\text { Crustacés } \\
\text { Crevette } \\
\text { Tourteau } \\
\text { Homard }\end{array}$ & $\begin{array}{c}58 \\
30 \text { à } 100 \\
40 \text { à } 60 \\
\simeq 50 \\
\simeq 60 \\
40 \text { à } 80 \\
30 \\
30 \text { à } 80\end{array}$ & $\begin{array}{c}150 \\
60 \text { à } 150 \\
60 \\
80 \text { à } 140 \\
80 \text { à } 100 \\
35 \text { à } 60 \\
25 \text { à } 45 \\
10 \text { à } 245 \\
105 \\
6 \text { à } 15 \\
100 \\
200 \text { à } 340\end{array}$ & $\begin{array}{l}{[6]} \\
{[7-12-51]} \\
{[10]} \\
{[6-10-11-21]} \\
{[11]} \\
{[10]} \\
{[7-10-44]} \\
{[6-21]} \\
{[6]} \\
{[6-10]} \\
{[44]} \\
{[7-44]}\end{array}$ \\
\hline
\end{tabular}

\subsection{Transferts par la nourriture (tableau V)}

Les études de transfert du Tc par la nourriture consistent à fournir à une espèce un ou plusieurs repas contaminés par du ${ }^{99 \mathrm{~m}} \mathrm{Tc}$ et à suivre son épuration en eau de mer renouvelée régulièrement avec un apport de nourriture identique mais non contaminée. Pour la plupart des espèces marines les régimes alimentaires ne sont pas connus, aussi est-il difficile d'établir un protocole expérimental proche des conditions naturelles. 
G. APROSI, M. MASSON

TABLEAU V

Période biologique $\left(T_{p}\right)$ du technétium pour quelques espèces marines benthiques et fraction éliminée $\%$ correspondante (la voie de contamination est la nourriture)

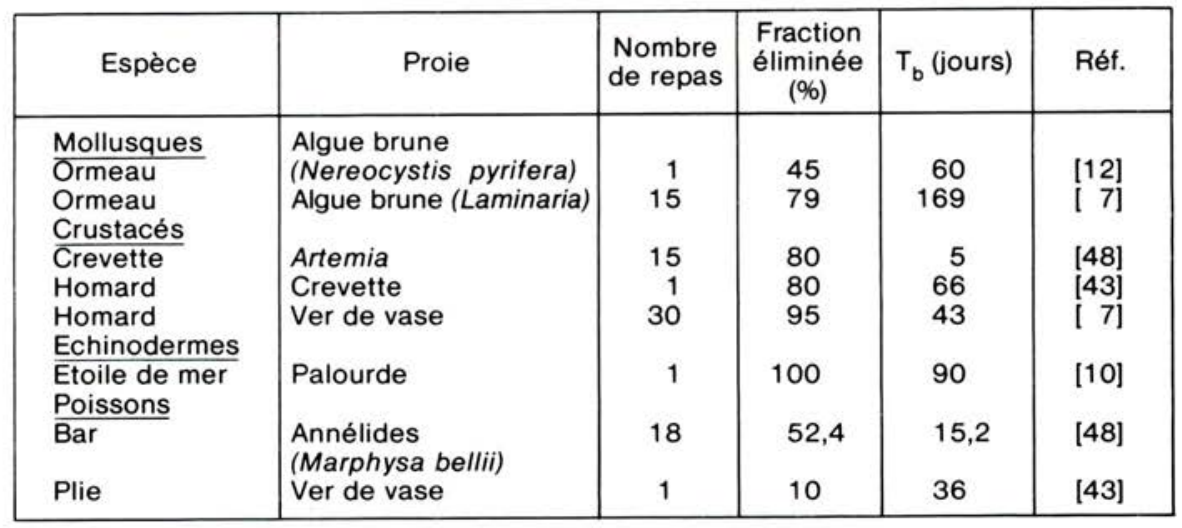

Les expériences de contamination par la nourriture ont montré que le taux d'assimilation du technétium par les organismes (poisson - la blennie et crevette - Palaemon elegans) est, en général, de $20 \%$ de l'activité totale des repas $[43,48]$.

\subsection{Transferts par le sédiment}

$\mathrm{Ce}$ transfert concerne les espèces benthiques susceptibles de se contaminer à partir du sédiment auxquelles elles sont inféodées.

\section{TABLEAU VI}

Facteur de transfert du technétium dans des organismes benthiques contaminés expérimentalement à partir du sédiment

\begin{tabular}{|c|c|c|c|c|c|}
\hline Organisme & $\begin{array}{c}\text { Nombre } \\
\text { d'individus }\end{array}$ & $\begin{array}{c}\text { Durée } \\
\text { essai } \\
\text { (jours) }\end{array}$ & $\begin{array}{c}\text { Etat de } \\
\text { valence }\end{array}$ & FT & Référence \\
\hline $\begin{array}{c}\text { Palourde } \\
\text { " }\end{array}$ & 5 & 36 & +7 & 0,07 à 0,19 & {$[10]$} \\
Ver de sable & 4 & 4 & +4 & 0 à 0,22 & {$[10]$} \\
Ver de vase & 5 & 35 & +7 & 0,5 & {$[38]$} \\
activité/g organismes & 15 & 35 & +7 & 0,5 & {$[38]$} \\
FT $=\frac{15}{\text { activités/g sédiment }}$ & & & & & \\
\hline
\end{tabular}

Seules, deux expériences ont été effectuées dans ce domaine. Le tableau VI en donne les résultats principaux; les facteurs de transfert sédiment-espèces sont faibles.

L'épuration des espèces est en général très lente. Lorsque des palourdes contaminées sont placées en milieu inactif après 2 mois et demi de contamination par du sédiment marqué au ${ }^{95 \mathrm{~m}} \mathrm{Tc}$, seuls $40 \%$ du radioélément fixé sont perdus. Cette observation est valable que le technétium soit sous forme réduite ou sous forme de pertechnétate [10]. 


\section{RÉSULTATS DES ANALYSES DU ${ }^{90}$ TC DANS DES ÉCHANTILLONS PRÉLEVÉS IN SITU}

L'analyse radiochimique du ${ }^{99} \mathrm{Tc}$ a été effectuée dans des échantillons marins prélevés autour des usines de retraitement de La Hague et de Windscale. Les tableaux VII et VIII donnent les résultats de la concentration du ${ }^{99}$ Tc dans des algues et des organismes de la faune benthique prélevés au cap de La Hague (Fig. 1) et en mer d'Irlande.

TABLEAU VII

Concentration de ${ }^{99} \mathrm{TC}$ dans des algues et des organismes prélevés au cap de La Hague [31] (concentration moyenne de l'eau au large de La Hague : $0,5 \mathrm{pCi} / \mathrm{l}, 0,018 \mathrm{~Bq} / \mathrm{l})$

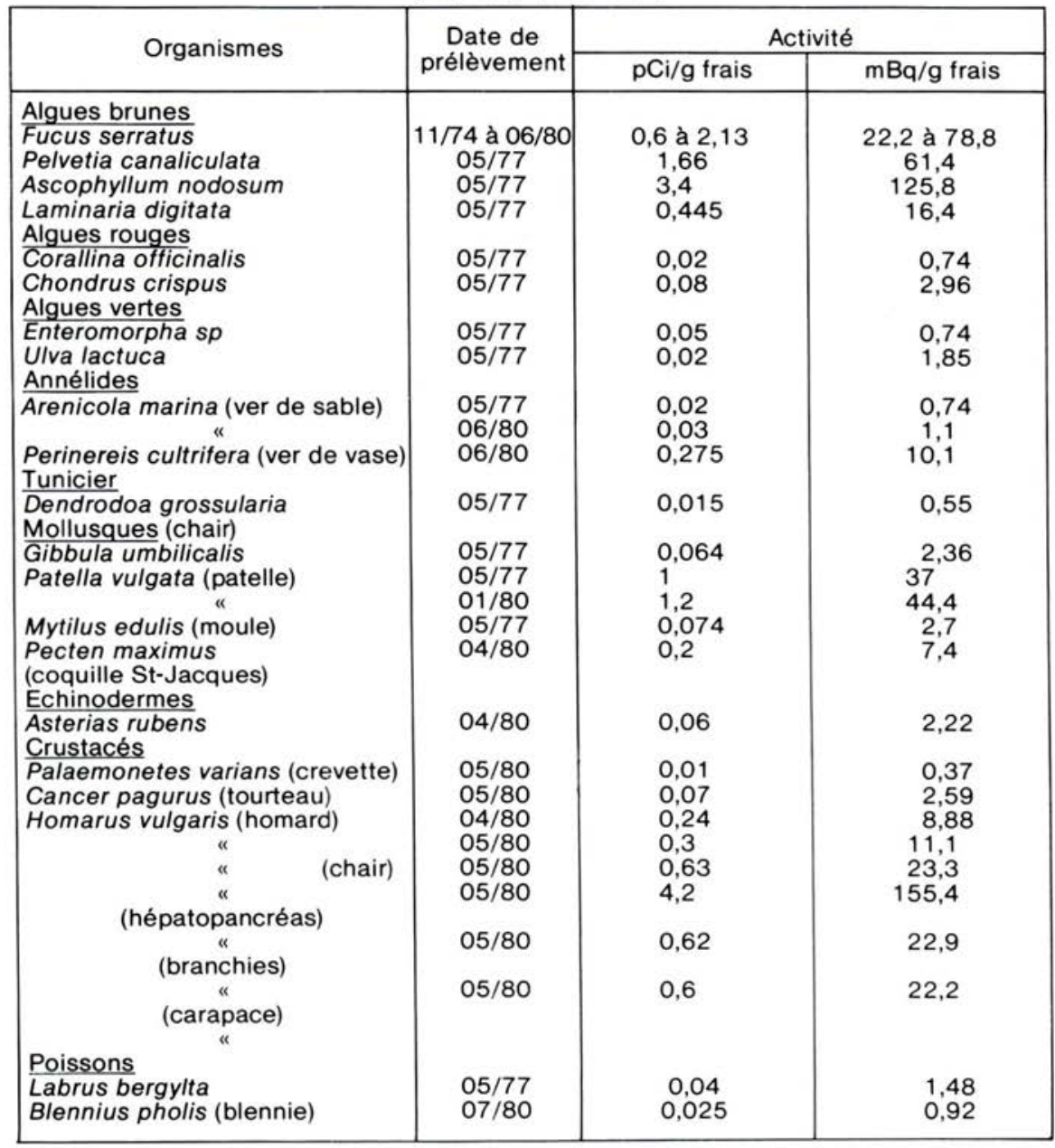




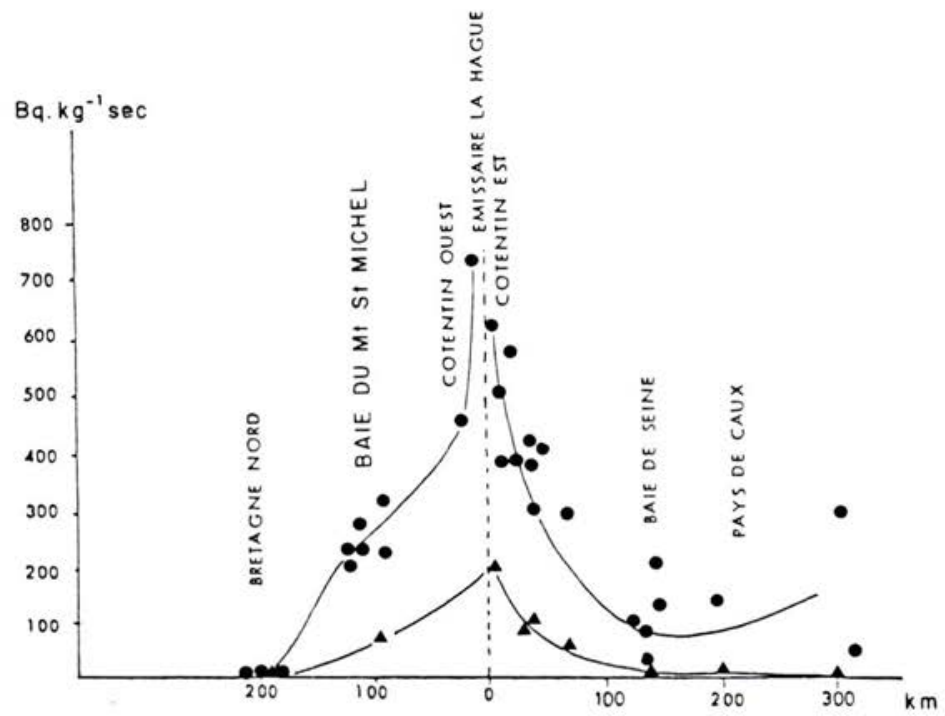

Fig. 1. - Répartition spatiale de ${ }^{99} \mathrm{Tc}$ de part et d'autre de l'émissaire de La Hague [39] - Fucus sp. (algue brune); $\boldsymbol{\Delta}$ Patella sp. (patelle $\gamma$ ).

A près de $100 \mathrm{~km}$ de part et d'autre des émissaires, on retrouve du Tc en quantités mesurables. Le technétium se caractérise donc par une capacité de diffusion très grande dans le milieu $[46,48]$.

TABLEAU VIII

Concentration de ${ }^{99} \mathrm{TC}$ dans des organismes prélevés dans l'environnement de l'usine de retraitement de Windscale (Sellafield) $[28,29,30,43]$

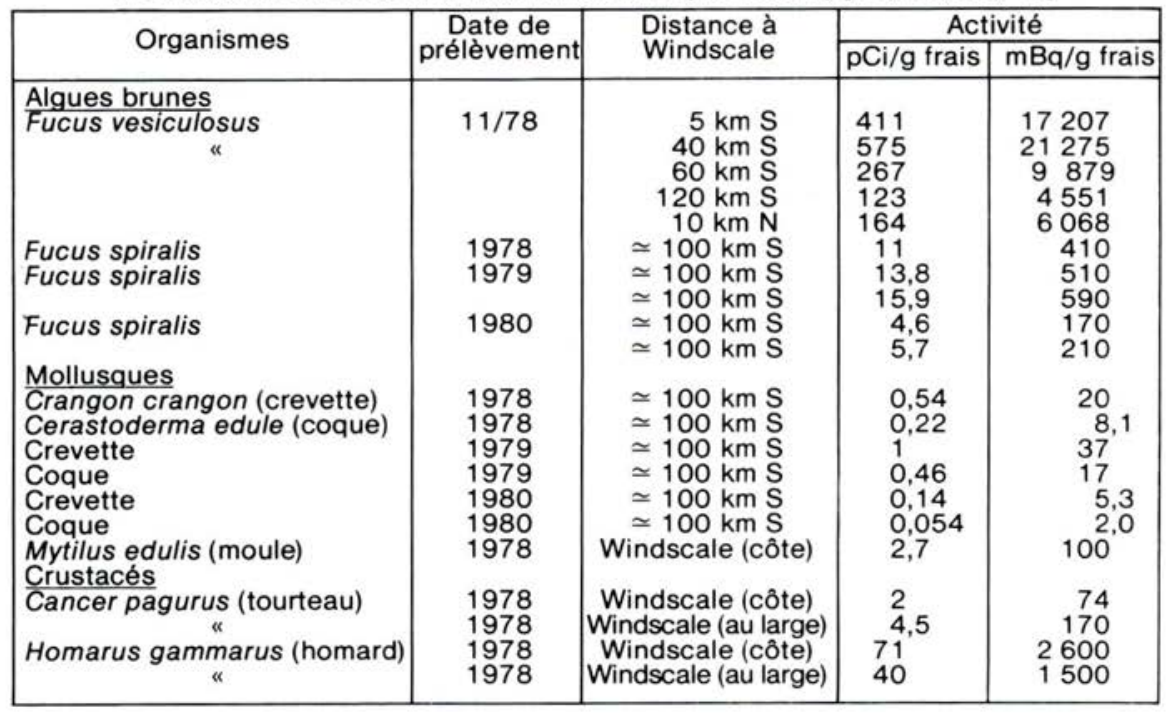


Les observations in situ confirment les résultats des mesures en laboratoire présentés précédemment : les organismes qui fixent le plus le technétium sont les Fucus et les Ascophyllum, les homards, les annélides Perinereis Cultrifera (ver de vase) et les mollusques (patelles). La comparaison des résultats entre La Hague et Windscale montre des différences de l'ordre de $10^{2}$ et quelquefois beaucoup plus, c'est le cas du homard où il a été observé des valeurs de $1500 \mathrm{mBq} / \mathrm{g}$ (frais) à Windscale pour $9 \mathrm{mBq} / \mathrm{g}$ (frais) à La Hague. II faut noter que dans l'eau de mer les quantités de technétium varient énormément en fonction des rejets et des conditions du milieu. Les rejets à Windscale varient entre 20 et $1000 \mathrm{mBq} / \mathrm{I}$; à La Hague, l'activité moyenne est de $18 \mathrm{mBq} / \mathrm{l}$ d'après Patti et Masson (non publié).

Notons, enfin, que dans le sable de la mer d'Irlande, à $100 \mathrm{~km}$ au sud de l'émissaire, les concentrations en ${ }^{99} \mathrm{Tc}$ sont de : $3,7 \mathrm{~m} \mathrm{~Bq} / \mathrm{g}$ (1978), $4 \mathrm{mBq} / \mathrm{g}$ (1979), 1,2 mBq/g (1980). Ces activités paraissent importantes ; s'agit-il de sables très réducteurs?

\section{CONCLUSION}

Ce document a recensé l'ensemble des résultats publiés à ce jour et ayant pour objet le transfert du technétium à des sédiments et à des espèces marines benthiques. II y a seulement quelques années, la pauvreté des informations bibliographiques sur ce sujet $[3,27,51]$ avait incité un certain nombre de laboratoires à développer des programmes de recherche, ce qui nous permet aujourd'hui de tirer des informations des résultats obtenus.

\section{Résultats expérimentaux et in situ}

Les résultats des études in situ sont en concordance avec les études de laboratoire. Les espèces qui présentent in situ une grande affinité pour le technétium sont les mêmes qu'en milieu expérimental.

La forme stable et soluble du technétium dans un milieu de force ionique élevée comme le milieu marin bien oxygéné est le pertechnétate $\left(\mathrm{TCO}_{4}{ }^{-}\right.$, valence +7$)$.

De manière générale, les sédiments oxydants fixent peu ou pas le technétium ; les coefficients de distribution varient de 0 à 50. Par contre, un sédiment frais réducteur en milieu fermé (anoxie) le fixe fortement. Les coefficients de distribution peuvent atteindre 1000, et plus le rapport poids du sédiment/volume d'eau est grand, plus la fixation est importante. Alors qu'un sédiment oxydant désorbe rapidement le technétium avec une période de quelques jours, un sédiment réducteur le désorbera très lentement.

Les facteurs de concentration correspondant au transfert du technétium aux organismes à partir des sédiments et de l'eau sont le plus souvent faibles, inférieurs à 0,5 dans le cas des sédiments (très peu d'expériences portent sur ce thème) et inférieurs à 10 dans le cas de l'eau. Seuls parmi les espèces étudiées, les algues brunes, les vers de vase et le homard présentent des facteurs de concentration de 1000 et plus, la voie de contamination étant l'eau. Lorsque la voie de contamination est la nourriture, les organismes étudiés présentent un taux d'assimilation de $20 \%$ 
de l'activité totale ingérée. II faut signaler que pour la plupart des espèces marines, les régimes alimentaires ne sont pas connus et les résultats sont biaisés par le fait que l'on impose à l'animal une nourriture qui ne correspond pas à celle qu'il peut trouver dans la nature.

Un certain nombre d'organes jouent un rôle dans la rétention du technétium ; ce sont des organes régulateurs (foie, glande digestive...) ou épurateur (rein...) ; en revanche, la chair (muscle, parties comestibles) le retient peu.

L'élimination s'opère le plus souvent selon deux phases, quelle que soit la vitesse de contamination : une phase rapide avec une période biologique de quelques jours et une phase lente ; elles correspondent à l'épuration de compartiments différents.

Les études biochimiques montrent que le technétium présente une affinité pour les protéines. Les images autoradiographiques permettent de le localiser dans les cellules qui ont un rôle digestif ou épurateur comme les cellules de la glande digestive et les cellules de type rénal de la glande verte des crustacés.

Certains paramètres influencent la fixation du technétium par les espèces étudiées, la lumière chez les algues, la forme physico-chimique chez beaucoup d'organismes, mais il n'y a pas d'influence de la taille, de la salinité et de la température. II faut signaler, à ce propos, que les informations sont très fragmentaires et ne peuvent pas être généralisées.

\section{Applications}

Le technétium se caractérise par une très grande dispersion en mer liée au fait que le milieu marin est oxydant ; on le retrouve jusqu'à plus de $100 \mathrm{~km}$ des émissaires de rejets; le transport se fait par l'eau. La forte capacité de fixation du technétium en milieu réducteur et turbide constitue une information utile pour le stockage des déchets et le transfert dans les estuaires. La voie préférentielle de transfert aux différents maillons d'une chaîne trophique est l'eau. Les espèces pouvant être retenues comme bioindicatrices de pollution par le technétium sont les algues brunes (Fucus) et le homard qui ont des facteurs de concentration élevés et des périodes biologiques très faibles. Les parties comestibles des organismes étudiés retiennent peu le technétium ; seuls le foie et le rein ont des capacités de fixation très fortes pour ce radionucléide.

\section{RÉFÉRENCES BIBLIOGRAPHIQUES}

[1] ANCELLIN J., GUEGUENIAT P., GERMAIN P. Radioécologie marine. Etude du devenir des radionucléides rejetés en milieu marin et applications à la radioprotection. Paris : Eyrolles, 1979.

[2] ANDERS E. Radiochemistry of technetium. USAEC Report NAS-NS-3021. Springfield, Va: NTIS, 1960.

[3] APROSI G. Le technétium, informations générales et synthèse bibliographiques. Rapport EDF-DER-HE31-79.26, 1979.

[4] APROSI G., MASSON M. Méthodologie d'étude du transfert du technétium dans quelques organismes marins. Rapport EDF-DER-HE31-81.15, 1981. 
[5] APROSI G., MASSON M. Technétium : toxicité et action massique de l'isotope 99, transferts eau-sédiments et sédiments-annélides. Rapport EDF-DER-HE31-81.16, 1981.

[6] APROSI G., MASSON M. Approche expérimentale de l'étude du transfert du technétium à quelques espèces marines benthiques. Rapport EDF-DER-HE31-81.17, 1981.

[7] APROSI G., MASSON M. Les cinétiques d'élimination du technétium chez quelques espèces marines benthiques après accumulation à partir de l'eau et de la nourriture: influence de différents paramètres sur la fixation et l'élimination. Rapport EDFDER-HE31-83.06, 1983.

[8] ATTREP M., ENOCHS J.A., BROZ L.D. Atmospheric technetium-99. Environ. Sci. Technol. 1971, 5, 344-345.

[9] BEASLEY T.M., GONOR J.J. Biogeochemical studies of technetium in marine and estuarine ecosystems. Progress Report, July 1979 - June 1980, Oregon State University, USA, Report DOE/EV/10251, 1980.

[10] BEASLEY T.M. Biogeochemical studies of technetium in marine and estuarine ecosystems. Progress Report, July 1980 - July 1981. Oregon State University, USA, Report DOE/EV/10251-3, 1981.

[11] BEASLEY T.M., GONOR J.J., LORZ H.V. Technetium : Uptake, organ distribution and loss in the mussel, Mytilus californianus (Conrad) and the oyster Crassostrea gigas (Thunberg), Mar. Env. Res., 1982, 7, 103-116.

[12] BEASLEY T.M., LORZ H.V., GONOR J.J. Biokinetic behaviour of technetium in the red abalone Haliotis rufescens : a re-assessment, Hith. Phys., 1982, 43, 501-507.

[13] BIRKS J.L. Medical radionuclides in marine environments. Nature, 1975, 255, 621-622.

[14] BITTEL R. Le teCHN2TIUM et l'environnement. Radioprotection, 1980, 15, 141-146.

[15] BONDIETTI E.A., FRANCIS C.W. Geologic migration potential of technetium-99 and neptunium 237. Science, 1979, 203, 1337-1340.

[16] BURKHOLDER H.C., CLONINGER M.O., BAKER D.A., JANSEN G. Incentives for partitioning high-level waste. US Report BNWL-1927, Springfield, VA: NTIS USA, 1975.

[17] CASTELLVI J.W., AMENGUAL P. Etude du cycle du soufre dans l'eau et les sédiments du plateau continental. Rapp. Comm. Int. Mer. Medit., 1981, 27(3) 25-26.

[18] DUURSMA E.K., BOSCH C.J. Theoretical, experimental and field studies concerning diffusion of radioisotopes in sediments and suspended particles of sea. Part B. Methods and experimentals. Neth. J. Sea. Res., 1970, 4, 395-469.

[19] EHRHARDT K.C., ATTREP M. Technetium in the atmosphere. Environ. Sci. Technol., $1978,12,55-57$.

[20] FISCHER N.S. Bioaccumulation of technetium by marine phytoplancton. Environ. Sci. Technol., 1982, 16, 579-581.

[21] FOWLER S.W., BENAYOUN G., PARSI P., ESSA M.W.A. Experimental studies on the bioavailability of technetium in selected marine organisms (IAEA - SM $248 / 113)$ In: Impacts of radionuclide releases into the marine environment, Vienne, 6-10 Oct. 1980. Vienne : AIEA, 1981, 319-339.

[22] FOWLER S.W., ASTON S.R., BENAYOUN G., PARSI P. Bioavailability of technetium from artificially labelled North East Atlantic deep-sea sediments. Mar. Environ. Res. 1983, 8, 87-100.

[23] GEARING P., VAN BAALEN C. et PARKER P.L. Biochemical effects of Tc-99 pertechnetate on microorganisms. Plant. Physiol., 1975, 55, 240-246.

[24] GRASSI J. Etude électrochimique des équilibres redox du technétium dans différents milieux aqueux (en vue de l'exploitation en médecine nucléaire). Thèse Chimie Analytique, Université Paris VI, 23 nov. 1977. 
[25] GRIMWOOD P.D., WEBB G.A.M. Assessment of the radiological protection aspects of disposal of high level waste on the ocean floor. Report NRPB - R48, Harwell (UK).

[26] GROMOV V.V. Uptake of plutonium and other nuclear wastes by plankton. Mar. Sci. Comm., 1976, 2, 227-247.

[27] GROMOV V.V., SPITSYN V.I. Sorption of ${ }^{239} \mathrm{Pu},{ }^{106} \mathrm{Ru}$ and ${ }^{99} \mathrm{Tc}$ by bottom sediments of the Pacific ocean. Radiokhimiya, 1974, 16, 157-162.

[28] HUNT G.J. Radioactivity in surface and coastal water of the British Isles, 1978. Aquat. Environ. Monit. Rep. $n^{\circ} 4$ 1981, MAFF, Lowestoff.

[29] HUNT G.J. Radioactivity in surface and coastal water of the British Isles, 1979. Aquat. Environ. Monit. Rep. $n^{\circ} 6,1981$, MAFF, Lowestoft.

[30] HUNT G.J. Radioactivity in surface and coastal water of the British Isles, 1980. Aquat. Environ. Monit. Rep. $n^{\circ} 8,1982$. MAFF, Lowestoft.

[31] JEANMAIRE L., MASSON M., PATTI F., GERMAIN P., CAPELLINI L., Technetium 99 content in some marine organisms collected near La Hague, France. Mar. Poll. Bull., 1981, 12, 29-32.

[32] KENNA B.T. The search for technetium in nature. J. Chem. Ed. 1962, 39, 436-442.

[33] KOTEGOV K.V., PAVLOV O.N. et SHVEDOR V.P. Technetium. In : Advances in inorganic chemistry and radiochemistry. New-York: Academic Press, 1968.

[34] LAVRUKHINA A.K., POZDNYAKOV A.A. Technetium. In : Analytical chemistry of Tc, $\mathrm{Pm}$, At and $\mathrm{Fr}$ (A.P. Vinegraden, Ed.) Ann Arbor: Humphrey science, 1970.

[35] LEFORT M. Oxydo-réduction du couple $\mathrm{TCO}_{2}-\mathrm{TcO}_{4}$ en solutions diluées sous l'effet du rayonnement gamma. Bull. Soc. Chim. Fr., $1969,882$.

[36] LUYKX F. Technetium discharges into environment. Document n० 5457/80 (VIE/2). Luxembourg: Commission of the European Communities, 1980.

[37] MASSON M. Les sites de fixation du technétium dans quelques organismes marins Rapport EDF-DER-HE31-83.05, 1983.

[38] MASSON M., APROSI G., LANIECE A., GUEGUENIAT P., BELOT Y. Approches expérimentales de l'étude des transferts du technétium à des sédiments et à des espèces marines benthiques. (IAEA-SM-248/124). In : Impacts of radionuclide releases into the marine environment, Vienne, 6-10 Oct. Vienne : AIEA 1981, 341-359.

[39] MASSON M., PATTI F., CAPELLINI L., GERMAIN P., JEANMAIRE L. Etude de la dispersion du technétium -99 sur les cótes françaises de la Manche à l'aide de deux indicateurs biologiques: Fucus sp. et Patella sp. J. Radioanal. Chem., 1983, 77, 247-255.

[40] PAQUETTE J., REID J.A.K. et ROSINGER E.L.J. Le comportement du technétium en relation avec le stockage définitif des déchets nucléaires. Atomic Energy of Canada, Ontario. Dossier technique TE.25, 1980.

[41] PATTI F. Détermination du technétium -99 dans les eaux résiduaires radioactives. Rapport DPr-82/4-SPS, 1982.

[42] PATTI F., CAPELLINI L., JEANMAIRE L., MASSON M. Détermination du technétium 99 dans les échantillons biologiques marins (IAEA-SM-252/49). In : Methods of lowlevel counting and spectrometry, Berlin, 6-10 Avr. 1981, Vienne : AIEA, 1982.

[43] PENTREATH R.J., JEFFERIES D.F., LOVETT M.B., NELSON D.M. The behaviour of transuranic and other long-lived radionuclides in the Irish sea and its relevance to the deep sea disposal of radioactive wastes. In : Marine Radioecology, Tokyo. 1-5 Oct. 1979. Paris : OCDE, 1980, 203-220.

[44] PENTREATH R.J. The biological availability to marine organisms of transuranium and other long-lived nuclides (IAEA-SM-248/102). In: Impacts of radionuclide releases into the marine environment, Vienne, 6-10 Oct. 1980. Vienne: AIEA, 1981, 241-272. 
[45] SAAS A., DENARDI J.L., COLLE C., QUINAULT J.M. Cycle du molybdène et du technétium dans l'environnement : évolution physico-chimique et mobilité dans les sols et les végétaux. In : 2 . Symposium international de radioécologie, Cadarache, 19-22 juin 1979. Cadarache: CEA, 1980, 443-490.

[46] SCHULTE E.H., SCOPPA P., SECONDINI A. Comportamento del tecnezio nell' ambiente marino: mobilita dell'anione pertecnetato. 4. Convegno nazionale radiochimica e chimica nucleare, Padova, 1982, 185-194.

[47] SCHULTE E.H., SECONDINI A., SCOPPA P. Trasferimento del tecnezio attraverso le catene alimentari marine. 22. Congresso nazionale dell'Associazione italiano di protezione contro le radiazioni, Brescia, 23-26, giugno 1981, vol. 1, 563-570.

[48] SCOPPA P., SCHULTE E.H., SECONDINI A. Application of technetium $-95 \mathrm{~m}$ in experimental radioecology : chemical form and behaviour in the marine environment. In: Technetium in chemistry and nuclear medicine (DEUTSCHE., Ed.). Verona: Cortina, 1983, 219-226.

[49] SCOPPA P., SECONDINI A., SCHULTE E.H. Indagini sulla stabilita dell'anione pertecnetato nell'ambiente marino. 22. congresso nazionale dell'Associazone italiana di protezione contro le radiazioni, Brescia, 23-26 giugno 1981, vol. 1, 503-511.

[50] SHREINER F. FRIED S., FRIEDMAN A. Extended time observation of the diffusion of neptunyl and partechnetate ions in deep sea and reducing sediments ; short term diffusion of salinity ion. Subseabed disposal program annual report for 1982 . Sandia National Laboratory, report SAND - 0664/II, 1982, 815-824.

[51] SPIES R.B. Uptake of technetium from seawater by red abalone Haliotis rufescens. Hith. Phys., 1975, 29, 695-699.

[52] TOPCUOGLUS., FOWLERS.W. Biokinetics of technetium $\left({ }^{95 \mathrm{~m}} \mathrm{TC}\right)$ in marine macroalgae. ICSEM 28. Congress and plenary assembly, Cannes, 2-11 Dec. 1982. Rapp. Comm. int. Mer Medit., 1982, 28, (7) 257-259.

[53] WILDUNG R.E., Mc FADDEN K.M., GARLAND T.R. Technetium sources and behaviour in the environment. J. Environ. Qual., 1979, 8, 156-161. 